\title{
A highly efficient exact algorithm for the uncapacitated multiple allocation $p$-hub center problem
}

\author{
Nader Ghaffarinasab ${ }^{\mathbf{a}^{*}}$
}

${ }^{a}$ Department of Industrial Engineering, University of Tabriz, Tabriz, Iran

\begin{tabular}{l}
\hline C H R O N I C L E \\
\hline Article history: \\
Received October 10, 2019 \\
Received in revised format: \\
October 28, 2019 \\
Accepted December 25, 2019 \\
Available online \\
December 26, 2019 \\
\hline Keywords: \\
p-hub center problem \\
Time-sensitive transportation \\
Mathematical modeling \\
Benders decomposition
\end{tabular}
\begin{abstract}
A B S T R A C T
Globalization and increasing competition in global markets have forced businesses to provide a high level of service to their customers. Time-sensitive transportation systems which are used in transportation of perishable goods, express mail delivery, and emergency services are playing a very important role in this regard. This paper addresses the problem of uncapacitated multiple allocation $p$-hub center problem (UMA $p \mathrm{HCP}$ ) which is fundamental in proper functioning of time-sensitive transportation systems. A mixed-integer programming formulation is proposed for the problem and a highly efficient Benders decomposition algorithm is developed for solving it. The proposed algorithm is capable of solving large-scale instances of the problem to optimality in order of seconds.
\end{abstract}

\section{Introduction}

Hubs are key facilities that act as intermediate centers for switching, sorting, and consolidating the commodities in many-to-many transportation systems and telecommunication networks. Generally, direct shipping of these commodities is not an economical decision as establishing direct linkages between each origin-destination (O/D) pair is extremely costly. Therefore, smaller number of connections are built in hub networks and each connection carry large traffic volume which in turn makes it possible to exploit economies of scale in transportation costs, especially on the inter-hub connections.

The hub location problem (HLP) deals with locating the hub facilities in the network and determining the way the non-hub nodes are allocated to the established hubs and the pattern based on which the O/D flow are routed via the hub facilities in such a way that a specific objective function is optimized. Regarding way the non-hub nodes are allocated to the hubs, we have two main types of hub networks. If each non-hub node send and receive the associated traffic through exactly one hub, then we deal with a single allocation hub network. On the other hand, if there is no restriction on the number of hubs with which each non-hub node can communicate, then the resulting network is called a multiple allocation hub network. A typical multiple allocation hub network is depicted in Fig. 1. In this figure, circles show the non-hub nodes, whereas the triangles represent the hub facilities.

\footnotetext{
* Corresponding author.

E-mail address: ngnasab@tabrizu.ac.ir (N. Ghaffarinasab)

(C) 2020 by the authors; licensee Growing Science, Canada. doi: $10.5267 /$ j.dsl.2019.12.001
} 


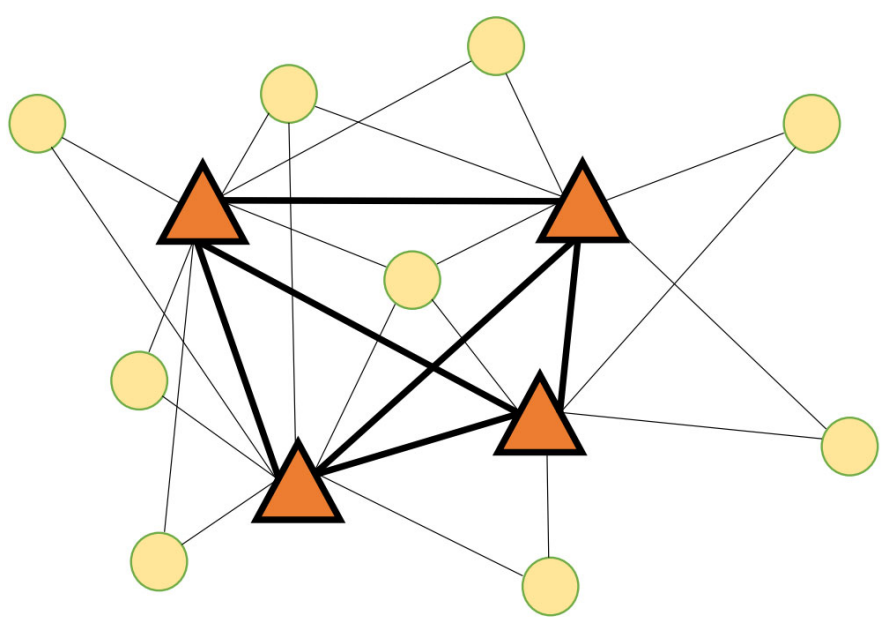

Fig. 1. Example of multiple allocation hub network

Most of the studies on the HLP deal with cost minimization objectives including fixed cost for establishing hubs and variable transportation costs. Although reducing cost is the primary concern in many practical problems in transportation, postal services, and telecommunication industries, it sometimes leads to unsatisfactory solutions in terms of service equitability as the distance between the $\mathrm{O} / \mathrm{D}$ pairs might be excessively large in the worst case. This is an undesirable outcome particularly when we deal with the delivery of perishable or time-sensitive items or when the aim is to provide a socially equitable service for different $\mathrm{O} / \mathrm{D}$ traffic which is essential for a sustainable transportation system. To remedy this drawback, one option is to use hub center problem in order to minimize the maximum distance (or cost) between the O/D pairs. In many situations in practice, the transported commodities are too sensitive to transportation time and need fast delivery. A possible real life application of this problem could be the location of hubs for transportation of perishable items (fruits, vegetables, seafood, etc.) where the hubs need to be located in a way that the clients could be served as soon as possible to avoid the damage of the products. Another application could be location of hubs in postal services where the express services are required by some customers for different reasons. In this work we address the uncapacitated multiple allocation $p$-hub center (UMA $p \mathrm{HCP}$ ). The goal is to locate a fixed number of hub facilities in such a way that the largest distances between the O/D pairs is minimized. The considered problem is modeled as mixed-integer linear program (MILP) and a highly efficient exact solution approach based on Benders decomposition is proposed to solve it. Extensive computational experiments are conducted to study the efficiency of the proposed solution algorithm and the effect of different input parameters on the optimal solutions of the problem.

The remainder of this paper is organized as follows. The literature review is presented in Section 2. A mathematical formulation for the problem is presented in Section 3. Section 4 describes the proposed Benders decomposition algorithm. Numerical results are presented in Section 5. Finally, Section 6 concludes the paper and provides some directions for future research.

\section{Background}

The HLP has become an important and well-studied area of research since 1980s within the field of facility location theory. The number of published studies on the HLP has had an increasing trend in recent years and different variants of the HLP have been studied so far. Recent surveys can be found in Alumur and Kara (2008), Campbell and O'Kelly (2012), and Farahani et al. (2013) for the interested readers. One of the important types of the HLP is the $p$-hub center problem which aims to serve the $\mathrm{O} / \mathrm{D}$ demand pairs by establishing a fixed number of $p$ hubs in such a way that the maximum transportation cost (time, distance, etc.) is minimized. This problem has many applications in transportation of time-sensitive commodities and hence it has been studied by numerous researches. Mixed integer programming (MIP) formulations for the single and multiple allocation $p$-hub center 
problem was proposed for the first time by Campbell (1994). Kara and Tansel (2000) developed three different integer linear programming (ILP) formulations of the single-allocation $p$-hub center problem, and found computationally that one of the formulations to be consistently superior. Kraticahttps://scholar.google.com/citations?user=PjOC2rkAAAAJ\&hl=en\&oi=sra and Stanimirović (2006) devised a genetic algorithm (GA) for the UMApHCP. Ernst et al. (2009) proposed a new ILP formulation for the uncapacitated single allocation $p$-hub center problem (USA $p$ HCP) using a new decision variable for the maximum collection and distribution cost/time between a hub and the associated O/D nodes. Sim et al. (2009) considered a $p$-hub center problem with normally distributed stochastic travel times and employed chance constraints to control the probability of the total travel time along a path to not exceed a given time bound. Meyer et al. (2009) presented a 2-phase algorithm for the USA $p \mathrm{HCP}$ where a set of potential optimal hub combinations was computed using a shortest path based branch and bound. The allocation phase was done using a reduced sized formulation giving the optimal solution. Furthermore, they developed a heuristic based on an ant colony optimization (ACO) approach to provide good upper bound for their algorithm. Bashiri et al. (2013) presented a GA based heuristic to solve the fuzzy capacitated $p$-hub center problem. Yang et al. (2013) developed a hybrid particle swarm optimization (PSO) algorithm for fuzzy $p$-hub center problem where the travel times were modeled using normal fuzzy vectors. Brimberg et al. (2017a) proposed a basic variable neighborhood search (VNS) heuristic for the UMApHCP. In another work, Brimberg et al. (2017b) proposed a general variable neighborhood search heuristic for the USA $p \mathrm{HCP}$.

Benders decomposition (Benders, 1962) is a method based on row generation which is suitable for solving MIP problems. In this procedure, the problem is reformulated by partitioning it into two simpler problems and a cutting plane approach is used to solve the reformulated problem. Benders decomposition algorithm has been successfully applied to the HLP in numerous research works. de Camargo et al. (2008) presented Benders decomposition algorithms for the uncapacitated multiple allocation hub location problem (UMAHLP). de Camargo et al. (2009a) developed a Benders decomposition algorithm for two versions of the uncapacitated multiple allocation HLP with flowdependent economies of scale. In another work, de Camargo et al. (2009b) proposed a generalized Benders decomposition algorithm for a multiple allocation HLP under congestion. Contreras et al. (2011a) developed a Benders decomposition algorithm for solving large-scale instances of the UMAHLP. Contreras et al. (2011b) applied a Benders algorithm for solving the stochastic uncapacitated hub location problem. de Camargo et al. (2011) proposed a hybrid algorithm combining the outer-approximation technique and a specialized version of Benders decomposition procedure for solving single allocation hub location problem under congestion. Gelareh and Nickel (2011) developed an accelerated Benders decomposition algorithm for an uncapacitated multiple allocation HLP tailored for urban transport and liner shipping network design problem. The authors extended the same Benders decomposition algorithm for the multi-period uncapacitated multiple assignment HLP under budget constraint in (Gelareh et al., 2015). Contreras et al. (2012) proposed a Benders algorithm for an extension of the classical capacitated multiple allocation HLP in which the amount of capacity installed at the hubs is a decision variable. Another Benders decomposition algorithm is devised for the manyto-many hub location routing problem by de Camargo et al. (2013). de Sa et al. (2013) proposed an accelerated Benders decomposition algorithm to solve the tree of hubs location problem. O'Kelly et al. (2015) presented a Benders decomposition algorithm for the HLP with price-sensitive demands. de Sa et al. (2015) proposed a Benders decomposition algorithm for the hub line location problem. Merakli and Yaman (2016) developed two Benders decomposition algorithms for solving large-scale instances of the robust uncapacitated multiple allocation $p$-hub median problem under polyhedral demand uncertainty. de Sa et al. (2018a) applied a Benders decomposition algorithm to the robust multiple allocation incomplete hub location problem where the $\mathrm{O} / \mathrm{D}$ demands and hub fixed costs were subject to uncertainty. Another Benders algorithm was devised for a similar problem with uncertain travel times and service time requirements by de Sa et al. (2018b). Ghaffarinasab and Kara (2019) developed Benders decomposition algorithms for solving two variants of the single allocation HLP, namely the uncapacitated single allocation hub location problem (USAHLP) and the uncapacitated single 
allocation $p$-hub median problem (USA $p H M P$ ). More recently, Taherkhani et al. (2019) devised a Benders algorithm to solve large-size instances of the profit maximizing hub location problems.

\section{Mathematical formulations}

Assume that $G=(N, A)$ is a graph with $N$ as the set of nodes and $A$ as the set of arcs such that $A=\{(i, j)$ : $i, j \in N, i<j\}$. Let $H \subseteq N$ represent the subset of candidate nodes for locating hubs. The number of hubs to be located is fixed and is denoted by $p$. For all $i, j \in N$, let $d_{i j}$ represent the distance between nodes $i$ and $j$. The total routing distance for the $\mathrm{O} / \mathrm{D}$ flow associated with pair $(i, j) \in A$ via hubs $k$ and $m$ in that order is calculated as:

$$
c_{i j k m}=d_{i k}+\alpha d_{k m}+d_{m j}
$$

where $\alpha$ is the discount factor to reflect economies of scale on the inter-hub connections $(0<\alpha<1)$, which can be interpreted as a speed-up (or cost decrease) factor incurred by usage of a faster (or more efficient) means of transport on such connections.

To develop an MIP formulation for uncapacitated multiple allocation $p$-hub center problem (UMA $p \mathrm{HCP}$ ), we use the binary variable $z_{k} \in\{0,1\}$ to be 1 if node $k$ is selected as a hub and 0 , otherwise. Also, the non-negative variable $x_{i j k m}$ denotes the fraction of traffic associated with the O/D pair $(i, j) \in A$, that is routed via hubs $k$ and $m$ in that order. Further, let $\beta$ represent the maximum distance between the $\mathrm{O} / \mathrm{D}$ pairs in the network. The problems consist of selecting $p$ nodes as hubs and determining how the O/D flows will be assigned to these hubs in such a way that the maximum distances between O/D pairs is minimized. The MIP model for the UMApHCP can be written as:

$$
\min \beta
$$

s.t:

$$
\begin{array}{ll}
\sum_{k \in H} z_{k}=p & \\
\sum_{k \in H} \sum_{m \in H} x_{i j k m}=1 & \forall(i, j) \in A \\
\sum_{m \in H} x_{i j k m}+\sum_{m \in H(m \neq k)} x_{i j m k} \leq z_{k} & \forall(i, j) \in A, k \in H \\
\beta \geq \sum_{k \in N} \sum_{m \in N} c_{i j k m} x_{i j k m} & \forall(i, j) \in A \\
z_{k} \in\{0,1\} & k \in H \\
x_{i j k m} \geq 0 & \forall(i, j) \in A, k, m \in H \\
\beta \geq 0 &
\end{array}
$$

The objective function (1) minimizes the maximum distances between O/D pairs. Constraint (2) determines the number of hubs to be located in the network. Constraints (3) assure that the whole flow associated with each O/D pair is routed via some hub pair. Constraints (4) state that the flows can only be routed via nodes that have been designated as hubs. Constraints (5) calculate the maximum distance between the O/D pairs. (6), (7), and (8) are the standard domain constraints for the decision variables.

\section{Benders reformulation}

Benders decomposition is a row generation based exact solution method that can be applied to solve large-scale mixed integer programming problems (Benders, 1962). In this technique, the problem is reformulated using a smaller number of variables and a large number of constraints. The problem is 
then solved using a cutting plane approach in which the relaxed problem, called as the master problem, is solved at each iteration by adding cutting planes found by the subproblem. Benders reformulation exploits the fact that computational burden of an MIP problem substantially increases with the problem size. Therefore, a divide-and-conquer scheme is employed to decompose a single large problem into smaller problems which can be solved more efficiently in terms of the computational time and resources. Motivated by this fact, in this work we apply Benders decomposition to the UMA $p$ HCP. In classical implementation of Benders algorithm, we need to solve the master problem at each iteration. However, we use a modern implementation within branch-and-cut framework where the master problem is solved in a single attempt and the cuts are added on the fly whenever required by utilizing recent developments in off-the-shelf solvers. We separate Benders cuts whenever a candidate integer solution is found in the branch-and-cut tree of the master problem. By doing so, the computational effort needed to solve an integer problem at each iteration is considerably reduced.

\subsection{The subproblem and the master problem}

By fixing the binary location variables vector as $\mathbf{z}=\hat{\mathbf{z}}$, the subproblem (SP) for the UMA $p$ HCP can be written as:

$\min \beta$

s.t:

$$
\begin{array}{ll}
\sum_{k \in H} \sum_{m \in H} x_{i j k m}=1 & \forall(i, j) \in A \\
\sum_{n \in H} x_{j / k n}+\sum_{m \in H(n \neq k)} x_{i j n k} \leq \hat{z}_{k} & \forall(i, j) \in A, k \in H \\
\beta \geq \sum_{k \in N} \sum_{m \in N} c_{i j k m} x_{i j k m} & \forall(i, j) \in A \\
x_{i j k m} \geq 0 & \forall(i, j) \in A, k, m \in H
\end{array}
$$

Let $\lambda_{i j}, \theta_{i j k}$, and $\mu_{i j}$ be the dual variable associated with constraints (10), (11), and (12) in the SP, respectively. Hence, the dual subproblem (DSP) for UMA $p$ HCP can be written as follows:

$$
\max \sum_{(i, j) \in A} \lambda_{i j}-\sum_{(i, j) \in A} \sum_{k \in H} \hat{z}_{k} \theta_{i j k}
$$

s.t:

$$
\begin{array}{ll}
\lambda_{i j}-\theta_{i j k}-\theta_{i j m}-c_{i j k m} \mu_{i j} \leq 0 & \forall(i, j) \in A, k, m \in H(k \neq m) \\
\lambda_{i j}-\theta_{i j k}-c_{i j k k} \mu_{i j} \leq 0 & \forall(i, j) \in A, k \in H \\
\sum_{(i, j) \in A} \mu_{i j} \leq 1 & \\
\mu_{i j} \geq 0 & \forall(i, j) \in A \\
\theta_{i j k} \geq 0 & \forall(i, j) \in A, k \in H \\
\lambda_{i j} \in \mathbb{R} & \forall(i, j) \in A
\end{array}
$$

We can now write the master problem (MP) for UMA $p \mathrm{HCP}$ as follows: 
$\min \omega$

s.t:

$$
\begin{array}{ll}
\omega \geq \sum_{(i, j) \in A} \lambda_{i j}^{t}-\sum_{(i, j) \in A} \sum_{k \in H} \theta_{i j k}^{t} z_{k} & t=1, \ldots, T \\
\sum_{k \in H} z_{k}=p & \\
z_{k} \in\{0,1\} & \forall k \in H
\end{array}
$$

in which $\left(\lambda^{t}, \boldsymbol{\theta}^{t}, \boldsymbol{\mu}^{t}\right)$ is the $t^{\text {th }}$ extreme point of the feasible solution space of the DSP. Observe that constraint (24) assures the installation of $p$ hubs in the network which in turn guarantees the feasibility of the SP at any iteration. Therefore, there is no need for adding feasibility cuts to the MP in our problems.

\subsection{Solving the dual subproblem}

In this section we devise algorithms for solving the DSP by inspection (without using standard solver) which resulting in substantial savings is the solution time of the problems. To this end, we first determine the optimal values of $\mu_{i j}$ variables. Due to the maximization sense in the objective function of the DSP (15)-(21) and according to constraints (18), we can conclude that the value of variable $\mu_{i j}$ associated with the O/D pair with longest distance will take 1 and the remaining variables will have 0 value in an optimal solution. We present a procedure for determining the optimal values of $\mu_{i j}$ variables in Algorithm 1.

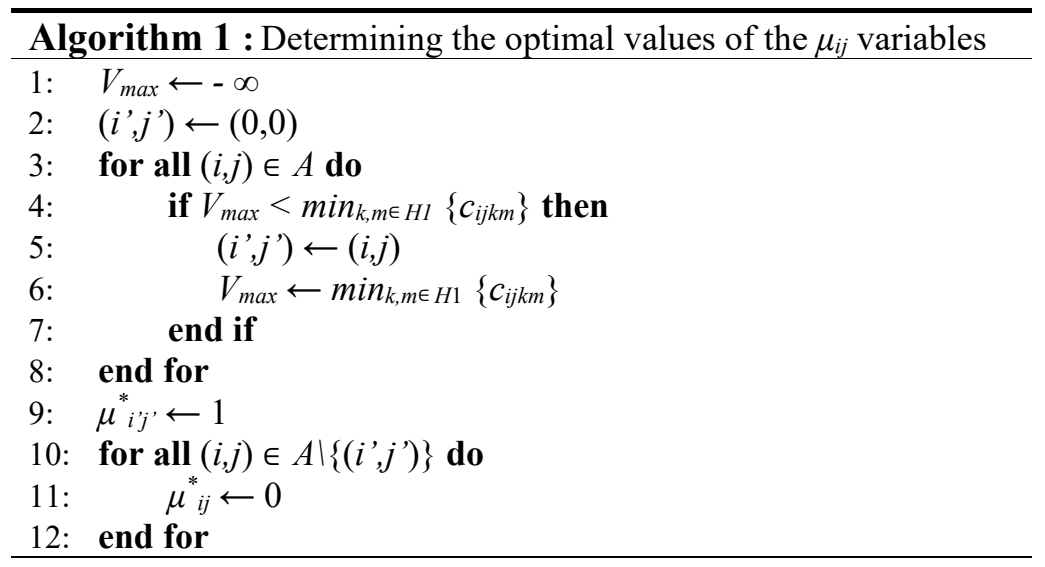

In the above algorithm $H 1$ is the set of opened hubs at the current iteration. Having obtained the optimal values for the $\mu_{i j}$ variables, the DSP for the UMA $p$ HCP can now be reduced to the following linear programming problem:

$$
\max \lambda_{i^{\prime} j^{\prime}}-\sum_{k \in H} \hat{z}_{k} \theta_{i^{\prime} j^{\prime} k}
$$

s.t:

$$
\begin{array}{ll}
\lambda_{i^{\prime} j^{\prime}}-\theta_{i^{\prime} j^{\prime} k}-\theta_{i^{\prime} j^{\prime} m} \leq c_{i^{\prime} j^{\prime} k m} & \forall k, m \in H(k \neq m) \\
\lambda_{i^{\prime} j^{\prime}}-\theta_{i^{\prime} j^{\prime} k} \leq c_{i^{\prime} j^{\prime} k k} & \forall k \in H \\
\theta_{i^{\prime} j^{\prime} k} \geq 0 & \forall k \in H \\
\lambda_{i^{\prime} j^{\prime}} \in \mathbb{R} &
\end{array}
$$


This problem can be solved by inspection and the corresponding optimal dual variables $\left(\lambda_{i}{ }^{\prime}{ }^{\prime}\right.$ and $\left.\theta_{i}{ }^{\prime}{ }^{\prime} k\right)$ can be determined using the complementary slackness conditions as explained by Contreras et al. (2011a). Furthermore, since for only one O/D pair (i.e., $\left.\left(i^{\prime}, j^{\prime}\right)\right)$ the values of the dual variables are nonzero, our master problem reduces to the following much smaller MIP which can be solved more efficiently.

$\min \omega$

s.t:

$$
\begin{array}{ll}
\omega \geq \lambda_{i^{\prime} j^{\prime}}^{t}-\sum_{k \in H} \theta_{i^{\prime} j^{\prime} k}^{t} z_{k} & t=1, \ldots, T \\
\sum_{k \in H} z_{k}=p & \\
z_{k} \in\{0,1\} & \forall k \in H
\end{array}
$$

\section{Computational experiments}

\subsection{Test problems}

We conduct an extensive set of computational experiments in order to demonstrate the efficiency of the proposed Benders decomposition algorithms. To this end, we use three well-known data sets from the hub location literature, namely the $\mathrm{CAB}$, the TR, and the AP data sets. The CAB data set constitute the airline passenger interactions data between 25 US cities in 1970 and is introduced by O'Kelly (1987). Our second data set is the TR data set (Tan and Kara, 2007) which is based on the cargo flows between 81 cities of Turkey. The third data set is the Australia Post (AP) data set which is introduced by Ernst and Krishnamoorthy (1996) and consists of 200 nodes representing postal districts in Sydney, Australia. In our experiments, we use instances with $|N| \in\{25,50,75,100,150,200\}$ from the AP data set. We set the values of the parameter $\alpha$ at four levels as: $\alpha \in\{0.2,0.4,0.6,0.8\}$. Furthermore, four values are used as the number of open hubs: $p \in\{2,3,4,5\}$. All the experiments have been run on a computer with Intel(R) Core(TM) i3-3220 CPU of $3.30 \mathrm{GHz}$ and $16 \mathrm{~GB}$ of RAM, using the Microsoft Windows 7 operating system. The proposed MIP model and the Benders decomposition algorithm are coded in JAVA and are solved by CPLEX version 12.6. We adopt a modern implementation of the Benders algorithm within a branch-and-cut framework, where the master problem is solved on a single tree and the optimality cuts are added one at a time using the lazy constraint callback function available in CPLEX. The time limit of two hours is used in our experiments.

\subsection{Numerical results}

Table 1 shows the results obtained by solving the UMApHCP with the CAB data set. The columns entitled $p$ and $\alpha$ denote the number of opened hubs and the value of the discount factor, respectively. The next two columns show the optimal objective function value and the corresponding set of opened hubs. The next columns show the solution times (in seconds) for the MIP model and the Benders decomposition algorithm.

The results reported in Table 1 show that the proposed Benders algorithm is able to obtain the optimal solution for all the instances of the $\mathrm{CAB}$ data set in very short computational time. The time needed to obtain the optimal solution by using the Benders algorithm is in order of hundredth of a second, whereas the average time taken by CPLEX to solve the MIP model is around 200 seconds. Note that for a given number of the opened hubs $p$, the optimal set of hub facilities change substantially as the value of discount factor varies. Observe also that for a fixed value of $p$, the optimal objective value increases as 
the value of the discount factor $(\alpha)$ increases. In contrast, for a fixed value of the discount factor, the value of the total transportation cost decreases as the number of opened hubs $(p)$ increases.

Table 1

Results for solving the problem for the CAB data set

\begin{tabular}{cccccc}
\hline \multirow{2}{*}{$p$} & $\alpha$ & \multirow{2}{*}{ Opt } & Hubs & MIP & CPU (s) \\
\cline { 5 - 6 } 2 & 0.2 & 2049.48 & 5,22 & 123.98 & 0.01 \\
& 0.4 & 2402.55 & 8,21 & 140.78 & 0.01 \\
& 0.6 & 2558.74 & 8,21 & 102.82 & 0.01 \\
& 0.8 & 2714.93 & 8,21 & 267.56 & 0.01 \\
\hline 3 & 0.2 & 1765.12 & $9,16,22$ & 194.44 & 0.02 \\
& 0.4 & 2064.67 & $5,12,23$ & 143.31 & 0.01 \\
& 0.6 & 2243.77 & $8,18,24$ & 106.23 & 0.01 \\
\hline 4 & 0.8 & 2515.58 & $8,17,24$ & 320.09 & 0.05 \\
& 0.2 & 1619.48 & $9,16,19,23$ & 286.13 & 0.04 \\
& 0.4 & 1774.45 & $9,12,16,23$ & 190.73 & 0.03 \\
& 0.6 & 2127.13 & $1,12,17,23$ & 158.95 & 0.02 \\
\hline 5 & 0.8 & 2437.71 & $3,6,8,24$ & 230.53 & 0.03 \\
& 0.2 & 1291.64 & $2,11,12,23,24$ & 257.19 & 0.04 \\
& 0.4 & 1599.74 & $11,12,18,23,24$ & 237.01 & 0.02 \\
& 0.6 & 1916.16 & $1,17,19,22,23$ & 190.68 & 0.03 \\
\hline
\end{tabular}

Table 2 shows the results obtained by solving the UMApHCP with the TR data set. As can be seen from the results, the proposed Benders algorithm has solved all the instances in quite short computational times. Note that the maximum solution times for different instances of the real-sized TR data set are around half a minute. Nevertheless, the MIP model could not be solved by CPLEX because of excessive memory requirements. Moreover, the changes in the optimal value of the objective function as a result of varying values for the input parameters $p$ and $\alpha$ are similar to the corresponding changes with the $\mathrm{CAB}$ data set.

\section{Table 2}

Results for solving the problem for the TR data set

\begin{tabular}{cccccc}
\hline \multirow{2}{*}{$p$} & $\alpha$ & \multirow{2}{*}{ Opt } & Hubs & \multicolumn{2}{c}{ CPU (s) } \\
\cline { 5 - 6 } & & & 26,62 & MIP & BD \\
\hline 2 & 0.2 & 1407.00 & 6,12 & Memory & 0.09 \\
& 0.4 & 1551.60 & 6,49 & Memory & 0.07 \\
& 0.6 & 1696.40 & 6,56 & Memory & 0.04 \\
\hline 3 & 0.8 & 1845.00 & $4,26,58$ & Memory & 0.78 \\
& 0.2 & 1261.60 & $3,12,54$ & Memory & 0.60 \\
& 0.4 & 1428.60 & $12,42,59$ & Memory & 0.31 \\
& 0.6 & 1596.00 & $4,6,73$ & Memory & 0.18 \\
\hline 4 & 0.8 & 1813.60 & $4,41,48,58$ & Memory & 6.19 \\
& 0.2 & 1130.40 & $3,49,59,60$ & Memory & 2.43 \\
& 0.4 & 1282.00 & $3,25,59,73$ & Memory & 0.50 \\
& 0.6 & 1474.40 & $25,34,64,73$ & Memory & 0.29 \\
\hline 5 & 0.8 & 1734.00 & $3,13,29,38,41$ & Memory & 36.31 \\
& 0.2 & 1025.00 & $6,24,59,64,72$ & Memory & 8.55 \\
& 0.4 & 1197.00 & $3,12,24,30,59$ & Memory & 1.95 \\
& 0.6 & 1430.40 & $25,30,56,59,64$ & Memory & 0.33 \\
\hline
\end{tabular}

Table 3 presents the results of solving the problem for the AP data set with small instancs $(|N|=25$ and 50). It can be seen from this table that the Benders algorithm all the instances in less than one second of CPU time. The MIP model could solve th instances with $|N|=25$ using CPLEX in average time of around 220 seconds. Note that for the problem with $|N|=50$ only one of the instances could be solved 
by CPLEX within the allowed time of two hours. For the remaining instances which was not solved within the two hours limit, the gap percentage between the corresponding upper and lower bounds are reported inside the parentheses.

Table 3

Results for solving the problem for the AP data set (small instances)

\begin{tabular}{|c|c|c|c|c|c|c|}
\hline \multirow{2}{*}{$|N|$} & \multirow{2}{*}{$p$} & \multirow{2}{*}{$\alpha$} & \multirow{2}{*}{ Opt } & \multirow{2}{*}{ Hubs } & \multicolumn{2}{|c|}{ CPU (s) } \\
\hline & & & & & MIP & $\mathrm{BD}$ \\
\hline \multirow[t]{16}{*}{25} & \multirow[t]{4}{*}{2} & 0.2 & 45813.71 & 4,16 & 116.64 & 0.02 \\
\hline & & 0.4 & 47336.19 & 4,16 & 122.84 & 0.01 \\
\hline & & 0.6 & 49123.94 & 5,12 & 66.69 & 0.01 \\
\hline & & 0.8 & 53112.15 & 5,12 & 36.37 & 0.01 \\
\hline & \multirow[t]{4}{*}{3} & 0.2 & 40780.89 & $5,7,17$ & 289.92 & 0.03 \\
\hline & & 0.4 & 41390.96 & $5,8,11$ & 179.59 & 0.03 \\
\hline & & 0.6 & 43001.19 & $5,7,16$ & 153.61 & 0.01 \\
\hline & & 0.8 & 48589.33 & $5,9,21$ & 32.37 & 0.01 \\
\hline & \multirow[t]{4}{*}{4} & 0.2 & 33019.26 & $2,5,9,16$ & 181.37 & 0.04 \\
\hline & & 0.4 & 36553.23 & $2,5,9,22$ & 306.88 & 0.04 \\
\hline & & 0.6 & 38746.77 & $5,7,18,21$ & 300.53 & 0.01 \\
\hline & & 0.8 & 48589.33 & $5,8,9,21$ & 141.27 & 0.01 \\
\hline & \multirow[t]{4}{*}{5} & 0.2 & 30218.49 & $2,5,14,16,21$ & 398.37 & 0.07 \\
\hline & & 0.4 & 32335.62 & $2,5,13,16,21$ & 280.12 & 0.03 \\
\hline & & 0.6 & 36682.65 & $5,7,13,21,22$ & 832.63 & 0.04 \\
\hline & & 0.8 & 48589.33 & $5,11,12,16,21$ & 122.65 & 0.01 \\
\hline \multirow[t]{16}{*}{50} & \multirow[t]{4}{*}{2} & 0.2 & 56118.40 & 7,12 & $2 \mathrm{~h}(18.3 \%)$ & 0.04 \\
\hline & & 0.4 & 57861.04 & 7,22 & $2 \mathrm{~h}(6.3 \%)$ & 0.05 \\
\hline & & 0.6 & 59549.30 & 7,31 & $2 \mathrm{~h}(2.8 \%)$ & 0.03 \\
\hline & & 0.8 & 61072.99 & 10,12 & $2 \mathrm{~h}(0.9 \%)$ & 0.03 \\
\hline & \multirow[t]{4}{*}{3} & 0.2 & 45698.26 & $4,8,44$ & $2 \mathrm{~h}(25.7 \%)$ & 0.09 \\
\hline & & 0.4 & 50870.54 & $1,8,31$ & $2 \mathrm{~h}(18.8 \%)$ & 0.11 \\
\hline & & 0.6 & 54337.55 & $3,8,31$ & $2 \mathrm{~h}(11.2 \%)$ & 0.10 \\
\hline & & 0.8 & 56061.85 & $10,14,42$ & $2 \mathrm{~h}(0.8 \%)$ & 0.04 \\
\hline & \multirow[t]{4}{*}{4} & 0.2 & 39390.22 & $4,10,27,31$ & $2 \mathrm{~h}(22.2 \%)$ & 0.14 \\
\hline & & 0.4 & 45575.81 & $4,9,27,31$ & $2 \mathrm{~h}(18.6 \%)$ & 0.18 \\
\hline & & 0.6 & 48412.31 & $1,10,15,42$ & $2 \mathrm{~h}(4.4 \%)$ & 0.06 \\
\hline & & 0.8 & 54489.92 & $6,10,33,41$ & $2 \mathrm{~h}(13.1 \%)$ & 0.06 \\
\hline & \multirow[t]{4}{*}{5} & 0.2 & 32648.59 & $1,4,9,28,31$ & $2 \mathrm{~h}(33.1 \%)$ & 0.17 \\
\hline & & 0.4 & 39975.05 & $1,4,9,25,42$ & $2 \mathrm{~h}(15.1 \%)$ & 0.23 \\
\hline & & 0.6 & 45784.24 & $1,4,10,18,42$ & $2 \mathrm{~h}(5.7 \%)$ & 0.07 \\
\hline & & 0.8 & 54088.39 & $1,10,14,41,42$ & 624.01 & 0.01 \\
\hline
\end{tabular}

The results obtained by solving the medium size AP instances $(|N|=75$ and 100) are presented in Table 4. While none of the instances could be solved as MIP model by CPLEX, the Benders algorithm could solve all the instances of medium sizes in less than three seconds. These results clearly demonstrate the high efficiency of the proposed solution algorithm.

Finally, Table 5 shows the results obtained by solving the large-scale AP instances $(|N|=150$ and 200). All the instances of size 150 are solved in less than 8 seconds. For the instances with 200 nodes, the maximum solution time is around 70 seconds. To the best of our knowledge, this is the first time that the instances of up to 200 nodes for the UMApHCP are solved to optimality in the literature. The solution times are extremely small for solving such large problem instance. This shows that even larger instances can be solved using the proposed exact solution algorithm in short computational times. Since the UMA $p$ HCP belongs to the class of NP-hard problems, proposing an exact solution algorithm which is able to solve large-scale instances of the problem in order of seconds is of utmost theoretical and practical importance. 
Table 4

Results for solving the problem for the AP data set (medium instances)

\begin{tabular}{|c|c|c|c|c|c|c|}
\hline \multirow{2}{*}{$|N|$} & \multirow{2}{*}{$p$} & \multirow{2}{*}{$\alpha$} & \multirow{2}{*}{ Opt } & \multirow{2}{*}{ Hubs } & \multicolumn{2}{|c|}{ CPU (s) } \\
\hline & & & & & MIP & $\mathrm{BD}$ \\
\hline \multirow[t]{16}{*}{75} & \multirow[t]{4}{*}{2} & 0.2 & 57634.34 & 9,64 & Memory & 0.05 \\
\hline & & 0.4 & 59523.34 & 10,17 & Memory & 0.04 \\
\hline & & 0.6 & 60294.07 & 10,32 & Memory & 0.04 \\
\hline & & 0.8 & 63270.38 & 10,46 & Memory & 0.03 \\
\hline & \multirow[t]{4}{*}{3} & 0.2 & 47001.38 & $7,11,33$ & Memory & 0.09 \\
\hline & & 0.4 & 51760.10 & $7,28,46$ & Memory & 0.12 \\
\hline & & 0.6 & 55423.99 & $5,11,46$ & Memory & 0.15 \\
\hline & & 0.8 & 58323.74 & $14,21,62$ & Memory & 0.04 \\
\hline & \multirow[t]{4}{*}{4} & 0.2 & 40597.89 & $7,12,39,46$ & Memory & 0.26 \\
\hline & & 0.4 & 47430.71 & $7,15,40,46$ & Memory & 0.21 \\
\hline & & 0.6 & 52444.66 & $4,14,23,46$ & Memory & 0.64 \\
\hline & & 0.8 & 56504.65 & $5,15,28,62$ & Memory & 0.06 \\
\hline & \multirow[t]{4}{*}{5} & 0.2 & 38145.40 & $2,7,12,40,46$ & Memory & 1.09 \\
\hline & & 0.4 & 43260.11 & $1,7,12,39,46$ & Memory & 0.62 \\
\hline & & 0.6 & 47867.04 & $1,7,13,38,62$ & Memory & 0.26 \\
\hline & & 0.8 & 55991.35 & $4,14,15,24,62$ & Memory & 0.06 \\
\hline \multirow[t]{16}{*}{100} & \multirow[t]{4}{*}{2} & 0.2 & 57617.15 & 13,85 & Memory & 0.26 \\
\hline & & 0.4 & 58983.15 & 14,23 & Memory & 0.25 \\
\hline & & 0.6 & 61204.64 & 15,23 & Memory & 0.09 \\
\hline & & 0.8 & 64357.63 & 17,23 & Memory & 0.09 \\
\hline & \multirow[t]{4}{*}{3} & 0.2 & 46419.59 & $5,16,62$ & Memory & 0.26 \\
\hline & & 0.4 & 51491.67 & $5,16,62$ & Memory & 0.48 \\
\hline & & 0.6 & 55704.65 & $5,16,61$ & Memory & 0.32 \\
\hline & & 0.8 & 58999.08 & $11,18,61$ & Memory & 0.17 \\
\hline & \multirow[t]{4}{*}{4} & 0.2 & 38879.25 & $5,17,61,77$ & Memory & 0.76 \\
\hline & & 0.4 & 44662.88 & $5,17,52,61$ & Memory & 1.14 \\
\hline & & 0.6 & 50520.56 & $5,18,31,61$ & Memory & 0.66 \\
\hline & & 0.8 & 57213.20 & $11,20,38,83$ & Memory & 0.17 \\
\hline & \multirow[t]{4}{*}{5} & 0.2 & 35659.13 & $5,17,37,61,68$ & Memory & 2.57 \\
\hline & & 0.4 & 41433.45 & $5,18,37,61,71$ & Memory & 1.54 \\
\hline & & 0.6 & 48224.01 & $5,19,26,35,83$ & Memory & 0.94 \\
\hline & & 0.8 & 55704.65 & $5,16,20,61,81$ & Memory & 0.17 \\
\hline
\end{tabular}

Table 5

Results for solving the problem for the AP data set (large instances)

\begin{tabular}{|c|c|c|c|c|c|c|}
\hline \multirow{2}{*}{$|N|$} & \multirow{2}{*}{$p$} & \multirow{2}{*}{$\alpha$} & \multirow{2}{*}{ Opt } & \multirow{2}{*}{ Hubs } & \multicolumn{2}{|c|}{ CPU (s) } \\
\hline & & & & & MIP & $\mathrm{BD}$ \\
\hline \multirow[t]{16}{*}{150} & \multirow[t]{4}{*}{2} & 0.2 & 60144.30 & 15,97 & Memory & 0.24 \\
\hline & & 0.4 & 62864.58 & 17,34 & Memory & 0.21 \\
\hline & & 0.6 & 63578.80 & 17,62 & Memory & 0.15 \\
\hline & & 0.8 & 66890.10 & 27,34 & Memory & 0.20 \\
\hline & \multirow[t]{4}{*}{3} & 0.2 & 48709.94 & $5,23,97$ & Memory & 0.57 \\
\hline & & 0.4 & 54348.48 & $10,24,64$ & Memory & 0.56 \\
\hline & & 0.6 & 59711.25 & $2,21,91$ & Memory & 0.48 \\
\hline & & 0.8 & 62375.67 & $27,38,122$ & Memory & 0.08 \\
\hline & \multirow[t]{4}{*}{4} & 0.2 & 39976.60 & $5,26,82,92$ & Memory & 1.35 \\
\hline & & 0.4 & 46407.19 & $5,26,82,92$ & Memory & 0.57 \\
\hline & & 0.6 & 53173.87 & $5,26,47,91$ & Memory & 0.57 \\
\hline & & 0.8 & 60622.67 & $26,29,38,123$ & Memory & 0.32 \\
\hline & \multirow[t]{4}{*}{5} & 0.2 & 38135.15 & $5,26,84,91,107$ & Memory & 7.65 \\
\hline & & 0.4 & 43117.74 & $5,26,68,86,91$ & Memory & 2.80 \\
\hline & & 0.6 & 50565.45 & $5,27,36,56,123$ & Memory & 1.41 \\
\hline & & 0.8 & 59526.00 & $5,21,29,91,121$ & Memory & 0.30 \\
\hline \multirow[t]{16}{*}{200} & \multirow[t]{4}{*}{2} & 0.2 & 61125.06 & 25,133 & Memory & 0.41 \\
\hline & & 0.4 & 63317.95 & 25,124 & Memory & 0.46 \\
\hline & & 0.6 & 64575.37 & 27,84 & Memory & 0.18 \\
\hline & & 0.8 & 67717.87 & 26,166 & Memory & 0.12 \\
\hline & \multirow[t]{4}{*}{3} & 0.2 & 49344.24 & $7,34,127$ & Memory & 1.40 \\
\hline & & 0.4 & 54636.74 & $10,34,87$ & Memory & 1.37 \\
\hline & & 0.6 & 60418.68 & $7,32,124$ & Memory & 2.22 \\
\hline & & 0.8 & 62991.73 & $37,53,165$ & Memory & 0.28 \\
\hline & \multirow[t]{4}{*}{4} & 0.2 & 40209.73 & $7,35,104,122$ & Memory & 5.48 \\
\hline & & 0.4 & 47663.44 & $7,36,111,122$ & Memory & 4.93 \\
\hline & & 0.6 & 54258.45 & $7,36,104,121$ & Memory & 4.25 \\
\hline & & 0.8 & 61238.58 & $7,31,39,165$ & Memory & 0.63 \\
\hline & \multirow[t]{4}{*}{5} & 0.2 & 38177.47 & $7,40,73,122,199$ & Memory & 70.73 \\
\hline & & 0.4 & 44670.61 & $7,36,79,95,121$ & Memory & 15.32 \\
\hline & & 0.6 & 51979.46 & $7,36,88,111,165$ & Memory & 18.43 \\
\hline & & 0.8 & 60449.58 & $3,32,39,52,164$ & Memory & 1.25 \\
\hline
\end{tabular}




\section{Conclusions}

We considered the uncapacitated multiple allocation $p$-hub center problem (UMA $p \mathrm{HCP}$ ) and formulated the problem as linear mixed integer programming model. In order to solve large-scale instances of the problem, a highly efficient Benders decomposition algorithm was proposed. An extensive set of computational experiments were done in order to analyze the efficiency of the proposed solution algorithm and to study the effect of different input parameters on the final solutions of the model. Obtained results demonstrate the capability of the proposed exact algorithm to solve large-scale instances of the problem in short computational times for the first time in the literature. Since the $\mathrm{UMA} p \mathrm{HCP}$ belongs to the class of NP-hard problems, proposing an exact solution algorithm which is able to solve large-scale instances of the problem in order of seconds is of utmost theoretical and practical importance. An interesting line for further extension of this research is to consider some sources of uncertainty (such as transportation costs, distances, etc.) in the proposed model. Furthermore, one may relax the classical HLP assumptions such as complete inter-hub network or fixed flow-independent discount factor for representing the economies of scale in order to deal with more realistic real-world situations. Finally, the proposed solution algorithm can be applied to other variants of the hub location problem with service level considerations such as hub covering problems.

\section{References}

Alumur, S. \& Kara, B.Y. (2008). Network hub location problems: The state of the art. European Journal of Operational Research, 190, 1-21.

Bashiri, M., Mirzaei, M., \& Randall, M. (2013). Modeling fuzzy capacitated $p$-hub center problem and a genetic algorithm solution. Applied Mathematical Modelling, 37(5), 3513-3525.

Benders, J.F. (1962). Partitioning procedures for solving mixed variables programming problems. Numerisch Mathematik, 4(1), 238-252.

Brimberg, J., Mladenovic, N., Todosijevic, R., \& Urosevic, D. (2017a). A basic variable neighborhood search heuristic for the uncapacitated multiple allocation $p$-hub center problem. Optimization Letters, 11(2), 313-327.

Brimberg, J., Mladenovic, N., Todosijevic, R., \& Urosevic, D. (2017b). General variable neighborhood search for the uncapacitated single allocation $p$-hub center problem. Optimization Letters, 11(2), 377388.

de Camargo, R.S., de Miranda Jr., G. \& Luna, H.P. (2008). Benders decomposition for the uncapacitated multiple allocation hub location problem. Computers \& Operations Research, 35(4), 1047-1064.

de Camargo, R.S., de Miranda Jr., G., Ferreira, R.P.M. \& Luna, H.P. (2009a). Multiple allocation hub-andspoke network design under hub congestion. Computers \& Operations Research, 36(12), 3097-3106.

de Camargo, R.S., de Miranda Jr., G. \& Luna, H.P. (2009b). Benders decomposition for hub location problems with economies of scale. Transportation Science, 43(1), 86-97.

de Camargo, R.S., de Miranda Jr., G. \& Ferreira, R.P. (2011). A hybrid outer-approximation/benders decomposition algorithm for the single allocation hub location problem under congestion. Operations Research Letters, 39(5), 329-337.

de Camargo, R.S., de Miranda Jr., G. \& Løkketangen, A. (2013). A new formulation and an exact approach for the many-to-many hub location-routing problem. Applied Mathematical Modelling, 37(12-13), $7465-7480$.

Campbell, J. F. (1994). Integer programming formulations of discrete hub location problems. European Journal of Operational Research, 72(2), 387-405.

Campbell, J.F. \& O'Kelly, M.E. (2012). Twenty-five years of hub location research. Transportation Science, 46, 153-169.

Contreras, I., Cordeau, J.F. \& Laporte, G. (2011a). Benders decomposition for large-scale uncapacitated hub location. Operations Research, 59(6), 1477-1490.

Contreras, I., Cordeau, J.F. \& Laporte, G. (2011b). Stochastic uncapacitated hub location problem. European Journal of Operational Research, 212(3), 518-528.

Contreras, I., Cordeau, J.F. \& Laporte, G. (2012). Exact solution of large-scale hub location problems with multiple capacity levels. Transportation Science, 46(4), 439-459. 
Ernst, A.T. \& Krishnamoorthy, M. (1996). Efficient algorithms for the uncapacitated single allocation $p$ hub median problem. Location Science, 4(3), 139-154.

Ernst, A.T., Hamacher, H., Jiang, H., Krishnamoorthy, M. \& Woeginger, G. (2009). Uncapacitated single and multiple allocation $p$-hub center problems. Computers \& Operations Research, 36, 2230-2241.

Farahani, R.Z., Hekmatfar, M., Arabani, A.B. \& Nikbakhsh, E. (2013). Hub location problems: A review of models, classification, solution techniques, and applications. Computers \& Industrial Engineering, 64(4) $1096-1109$.

Gelareh, S. \& Nickel, S. (2011). Hub location problems in transportation networks. Transportation Research Part E: Logistics and Transportation Review, 47(6), 1092-1111.

Gelareh, S., Monemi, R.N. \& Nickel, S. (2015). Multi-period hub location problems in transportation. Transportation Research Part E: Logistics and Transportation Review, 75, 67 - 94.

Ghaffarinasab, N. \& Kara, B.Y. (2019). Benders decomposition algorithms for two variants of the single allocation hub location problem. Networks and Spatial Economics, 19(1), 83-108.

Kara, B.Y. \& Tansel, B.C. (2000). On the single-assignment $p$-hub center problem. European Journal of Operational Research, 125, 648-655.

Kratica, J., \& Stanimirović, Z. (2006). Solving the uncapacitated multiple allocation $p$-hub center problem by genetic algorithm. Asia-Pacific Journal of Operational Research, 23(04), 425-437.

Merakl1, M. \& Yaman, H. (2016). Robust intermodal hub location under polyhedral demand uncertainty. Transportation Research Part B: Methodological, 86, 66 - 85.

Meyer, T., Ernst, A. T., \& Krishnamoorthy, M. (2009). A 2-phase algorithm for solving the single allocation p-hub center problem. Computers \& Operations Research, 36(12), 3143-3151.

O'Kelly, M.E. (1987). A quadratic integer program for the location of interacting hub facilities. European Journal of Operational Research, 32(3), 393-404.

O'Kelly, M.E., Luna, H.P.L., de Camargo, R.S. \& de Miranda Jr., G. (2015). Hub location problems with price sensitive demands. Networks and Spatial Economics, 15(4), 917-945

de Sa, E.M., de Camargo, R.S. \& de Miranda Jr., G. (2013). An improved benders decomposition algorithm for the tree of hubs location problem. European Journal of Operational Research, 226(2), 185-202.

de Sa, E.M., Contreras, I., Cordeau, J.F., de Camargo, R.S. \& de Miranda Jr., G. (2015). The hub line location problem. Transportation Science, 49(3), 500-518.

de Sa, E.M., Morabito, R. \& de Camargo, R.S. (2018a). Benders decomposition applied to a robust multiple allocation incomplete hub location problem. Computers \& Operations Research, 89, 31-50.

de Sa, E.M., Morabito, R. \& de Camargo, R.S. (2018b). Efficient benders decomposition algorithms for the robust multiple allocation incomplete hub location problem with service time requirements. Expert Systems with Applications, 93, 50-61.

Sim, T., Lowe, T.J. \& Thomas, B.W. (2009). The stochastic $p$-hub center problem with service-level constraints. Computers \& Operations Research, 36(12), 3166 - 3177.

Taherkhani, G., Alumur, S.A. \& Hosseini, S.M. (2019). Benders decomposition for profit maximizing hub location problems with capacity allocation.

Tan, P.Z. \& Kara, B.Y. (2007). A hub covering model for cargo delivery systems. Networks, 49(1), 28-39.

Yang, K., Liu, Y., \& Yang, G. (2013). An improved hybrid particle swarm optimization algorithm for fuzzy p-hub center problem. Computers \& Industrial Engineering, 64(1), 133-142.

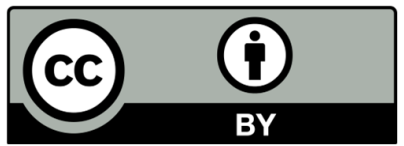

(C) 2020 by the authors; licensee Growing Science, Canada. This is an open access article distributed under the terms and conditions of the Creative Commons Attribution (CC-BY) license (http://creativecommons.org/licenses/by/4.0/). 